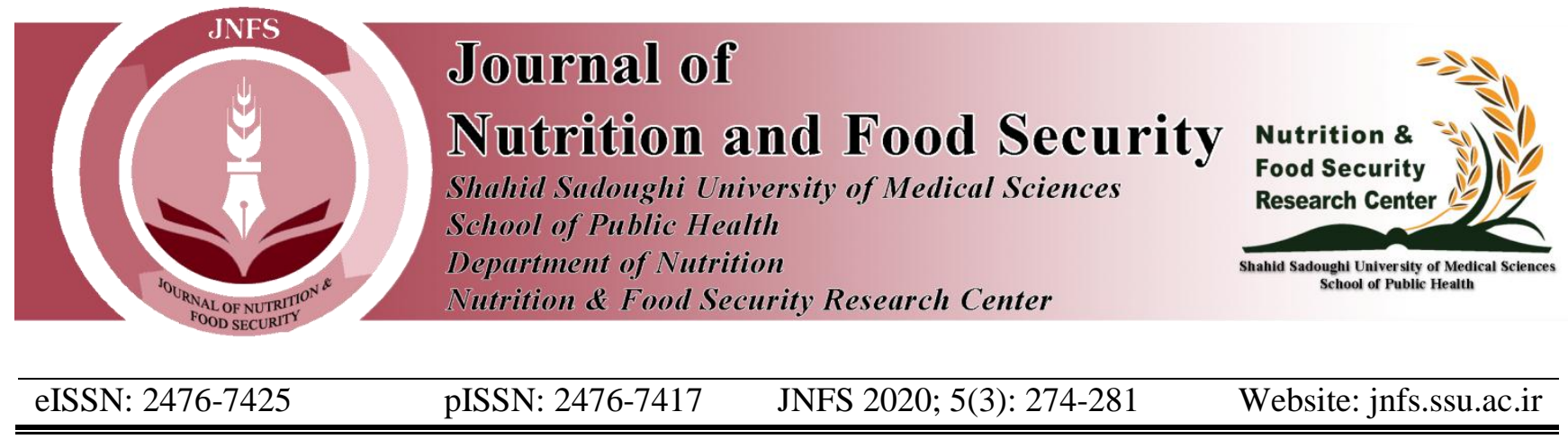

\title{
Effective Factors on Acute Malnutrition in Children Aged 6 to 59 Months in the Centre-West Region of Burkina Faso
}

Jeanne d'Arc Kabre; MSc ${ }^{1}$, Sibiri Bougma; MSc ${ }^{1}$, Bassibila Zoungrana; MSc ${ }^{1}$, Yves Traoré; PhD $^{1}$ \& Aly Savadogo; PhD $^{* 1}$

${ }^{1}$ Laboratory of Applied Biochemistry and Immunology (LaBIA), University Joseph KI-ZERBO, 03 BP 7021 Ouagadougou 03, Burkina Faso.

\begin{tabular}{l}
\hline ARTICLE INFO \\
\hline ORIGINAL ARTICLE \\
\hline Article history: \\
Received: 24 Feb 2020 \\
Revised: 21 Jun 2020 \\
Accepted: 10 Jun 2020 \\
*Corresponding author: \\
alysavadogo@ gmail.com \\
Laboratory of Applied \\
Biochemistry and Immunology \\
(LaBIA), University Joseph \\
KI-ZERBO, 03 BP 7021 \\
Ouagadougou $03, \quad$ Burkina \\
Faso. \\
Postal code: $03 \quad$ BP 7021 \\
Ouagadougou 03 \\
Tel: +226-70 35 62 27
\end{tabular}

\begin{abstract}
Background: In Burkina Faso, malnutrition is a public health problem. Despite the significant drop in the prevalence of malnutrition since 2009 according to the Directorate of Nutrition, malnutrition is still a major concern with regard to its consequences. The objective of this study was to determine the factors associated with acute malnutrition in children aged 6 to 59 months in the Sabou Health District, Burkina Faso. Methods: This was a descriptive, cross-sectional study conducted among 343 children aged 6 to 59 months in Sabou Health District, Burkina Faso from August $1^{\text {st }}$ to $30^{\text {th }}, 2016$. Factors associated with acute malnutrition were dietary factors, family factors, environmental factors, and infectious factors determined using SPSS version 21.0 software. The difference was statistically significant for $P$-value of less than 0.05 . Results: The prevalence of global acute malnutrition in the district was $9.0 \%$, of which $2.6 \%$ was severe according to the Brachial Perimeter. Several factors were associated with acute malnutrition in children under 5 years of age in the Sabou health district. Indeed, morbidity was a factor associated with acute malnutrition in children with a P-value of 0.007 . The low dietary diversity experienced by food-insecure households was also associated with acute malnutrition in children. Infant and young child feeding practices, i.e., inadequate introduction of complementary foods and low dietary diversity to children were among the factors associated with nutritional status $(P=0.007)$. Among mothers, $87.5 \%$ had heard of malnutrition, $66.5 \%$ did not recognize the signs of malnourished in their children, and $92.4 \%$ were unaware of the consequences of malnutrition. Conclusion: Based on the results, initiatives and programmes should be established for women, the bearers of life, to enhance their empowerment and educate them with regard to the integral growth of children, the builders of tomorrow.
\end{abstract}

Keywords: Causes; Acute malnutrition; Household; Child; Burkina Faso

Introduction

$\mathrm{M}$ alnutrition is a major public health problem worldwide; it is serious enough to call into

question the sustainable development goals (Resnick et al. 2015), particularly in the 
developing countries. For millions of survivors, malnutrition causes after-effects in the form of infirmity, chronic vulnerability to disease, and intellectual disability that can affect the human life cycle (UNICEF, 2000).

At the global level, an estimated study conducted by UNICEF/WHO/WB showed that 161 million children under 5 years of age were stunted, 51 million were underweight for height, and 42 million were overweight; none of these children were growing up healthy (Smith and Haddad, 2014). Despite overall reduction in the number of undernourished people during 1992-2013, subSaharan Africa has remained the region with the highest prevalence of undernourishment (24.8\%) with more than 223 million undernourished people (Carletto et al., 2013).

Each year, 20 million infants are born with low birth weight (15.5\% of all births), 13 million of them had intrauterine growth retardation (Blanc and Wardlaw, 2005).

In Burkina Faso, the maternal and child health situation is worrying (Ministry of Health, 2014). More than one child out of six dies before the age of five, which is equal to 125,000 deaths each year (UNICEF Staff, 2011).

Malnutrition affects children in rural areas $(37 \%)$ than urban areas $(21 \%)$ (National Institute of Statistics and Demography (INSD ), 2010). According to the National Nutrition Survey (Ministry of Health, 2016), the global acute malnutrition in Burkina Faso has a prevalence of $7.6 \%$, of which $1.4 \%$ of the cases are severe. At the regional level, disparities in prevalence exist from one region to another.

The survey shows that approximately one third of children under the age of five (27.3\%) suffer from stunting, 7.4\% of whom are severely stunted.

A national prevalence of underweight of $19.2 \%$ was estimated in 2016, of which $3.7 \%$ is severe (Ministry of Health, 2016). The prevalence of underweight ranges from $14.1 \%$ in the Central region to $25.7 \%$ in the Eastern region.

Despite the efforts made by the government, a need for further research exists on the factors associated with acute malnutrition, especially in rural areas, where the highest rates are recorded. So, this study was conducted to investigate the factors associated with acute malnutrition in children aged 6 to 59 months in the Sabou health district. More specifically, we intended to estimate the prevalence of acute malnutrition and identify the factors associated with acute malnutrition such as dietary factors, family factors, environmental factors and infectious factors.

\section{Materials and Methods}

The study tried to investigate effective factors on the acute malnutrition in children aged 6-59 months. This research was carried out in the Sabou health district in Burkina Faso from August $1^{\text {st }}$ to $30^{\text {th }}, 2016$. After enumeration of the households, a random draw was made by ENA software to select 385 households out of a total of 21,158 to conduct the study. In these households, the mothers of 343 children aged 6 to 59 months were interviewed. The data were collected on a survey form designed for the study. The brachial perimeter was the anthropometric measure used to classify the nutritional status of children. On the survey form adopted for the study, we collected data on socio-demographic variables such as: age, morbidity, household environment, parents' marital status, as well as parents' economic and educational level.

The household food diversity score was calculated by counting the number of food groups consumed in the household over a 24-hour period. This dietary diversity score was constructed on the basis of a list of 16 food groups classified into 12 groups (Yousaf et al., 2018).

The food insecurity score was calculated from the responses to 9 questions in a standardized questionnaire on household experiences, following the method recommended by FANTA 4 (Food And Nutrition Technical Assistance) (Carletto et al., 2013). For each response, a score was assigned according to the frequency of the corresponding event experienced by the household in the previous month: 0 (never), 1 (rarely), 2 (sometimes), or 3 (often). 
The infant and young child feeding (IYF) indicators were also calculated according to the international recommendations. The indicators included early initiation of breastfeeding, continued breastfeeding at one year of age, introduction of complementary foods, minimum dietary diversity, minimum frequency of meals, and minimum acceptable feeding.

\section{Results}

Our study involved 343 children aged 6 to 59 months including $47.5 \%$ boys and $52.5 \%$ girls. In Sabou Health District, $72.9 \%$ of the households did not have a latrine, $63.8 \%$ did not have a garbage bin (Table 1), 21.8\% did not have potable water for consumption (Table 1), and more than half of the households (68.8\%) were highly food insecure; $28.8 \%$ and only $2.3 \%$ were at moderate and low levels of food insecurity, respectively (Table 1).

Mostly family polygamous (65.3\%), households in Sabou Health District lived mainly from agriculture $(89 \%)$, commerce $(6 \%)$, and cattle breeding (4\%). About $70.3 \%$ of the mothers had no income-generating activity and the level of education was low overall. In fact, $71.9 \%$ of the household heads and $77.6 \%$ of mothers had no education. The average household size was 15 persons and the average number of children per mother was 6 . Many mothers were still unaware of the effects of malnutrition. Although $87.5 \%$ of the mothers had heard of malnutrition, $66.5 \%$ had no evidence to recognize a malnourished child and about $92.4 \%$ of the mothers were unaware of the consequences of malnutrition on their children. In our study, the prevalence of global acute malnutrition was $9.0 \%$, of which $2.6 \%$ was severe (Figure 1).

In Sabou Health District $54.8 \%$ of the households had low dietary diversity (score $<4$ ), $36.4 \%$ had medium diversity, and only $8.7 \%$ had high dietary diversity (Figure 2).

Of 31 cases of malnourishment, $61.3 \%$ were boys and $38.7 \%$ were girls, although the sample included $47.5 \%$ boys and $52.5 \%$ girls. The majority of malnourished children $(77.4 \%)$ were between 6 and 23 months of age (Table 2).

Almost all mother of malnourished children was interviewed and the findings showed that $96.8 \%$ had suffered from some kind of illness two weeks preceding the survey. In this regard, $16.1 \%$ of the malnourished children had developed malaria, 29\% had suffered from diarrhoea, and $54.9 \%$ experienced a respiratory infection (Table 3).

The prevalence of malnourished children living in households with severe and low food insecurity were $80.6 \%$ and $61.3 \%$, respectively (Table 4).

The IYF practices among the malnourished children remained very unsatisfactory and could explain their nutritional status. According to the findings, $99.7 \%$ of these children benefited from breast milk, only $22.6 \%$ were exclusively breastfed until 6 months and $54.8 \%$ of these malnourished children benefited from colostrum. In addition, none of the malnourished children had consumed vitamin A-rich fruits and vegetables the day before the survey. For complementary feeding, no malnourished children aged 6-8 months received solid, semi-solid, or soft food the day before the survey. Only one malnourished child (3.2\%) aged 6-23 months consumed at least 4 food groups.

The findings showed that five factors were associated with acute malnutrition in children under five years of age: age morbidity, low dietary diversity of households, accompanied by high food insecurity, and poor IYF practices, namely inadequacy of complementary foods and low dietary diversity among children. 


\section{Table 1. Levels of household food safety and security}

\begin{tabular}{lcc}
\hline Variables & $\mathbf{N}$ & $\%$ \\
\hline Latrines & 105 & 27.1 \\
$\quad$ Yes & 280 & 72.9 \\
No & & \\
Refuse receptacle & 139 & 36.2 \\
$\quad$ Yes & 246 & 63.8 \\
No & & \\
Drinking water source & 301 & 78.2 \\
Yes & 84 & 21.8 \\
No & & \\
Level of household food security & 265 & 68.8 \\
Low & 111 & 28.8 \\
Medium & 9 & 2.4 \\
High & & \\
\hline
\end{tabular}

Table 2. Relationship of acute malnutrition with age and gender

\begin{tabular}{lccc}
\hline Variables & Number of malnourished children & Percentage & P-value \\
\hline Gender & & & \\
Male & 19 & 61.3 & 0.075 \\
Female & 12 & 38.7 & \\
Age (month) & & & \\
$6-23$ & 24 & 77.4 & 0.014 \\
$24-59$ & 7 & 22.6 & \\
\hline
\end{tabular}

Table 3. Relationship between acute malnutrition and morbidity

\begin{tabular}{lccc}
\hline Variables & Number of malnourished children & Percentage & P-value \\
\hline Malaria & 5 & 16.1 & 0.007 \\
Diarrhoea & 9 & 29.0 & \\
Respiratory infection & 17 & 54.9 & \\
\hline
\end{tabular}

\section{Table 4. Dietary factors associated with acute malnutrition}

\begin{tabular}{lccc}
\hline Variables & Number of malnourished children & Percentage & P-value \\
\hline Dietary diversity & 19 & & \\
Low & 12 & 61.3 & 0.002 \\
Average & 0 & 38.7 & \\
High & 25 & 0.0 & \\
Food insecurity & 6 & 80.6 & 0.006 \\
Severe & 0 & 19.4 & \\
Average & 0 & 0 & \\
Low & &
\end{tabular}



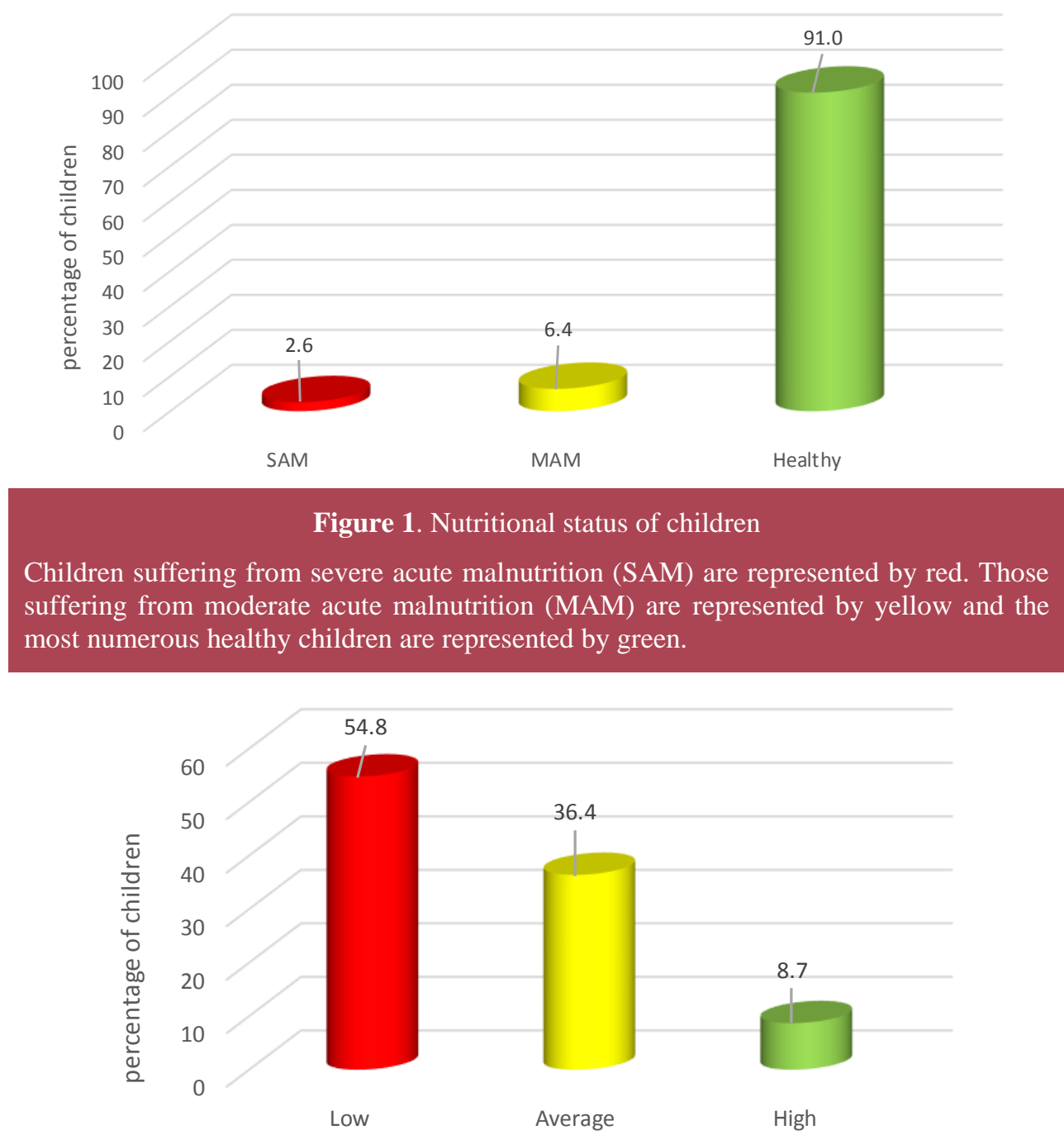

Figure 2. Distribution of households according to dietary diversity

The different households interviewed were distributed according to their dietary diversity. Thus, the maximum number of households (red) has a low dietary diversity and only less than $10 \%$ (green) has a high dietary diversity.

\section{Discussion}

The nutritional status of children under five years of age is often associated with certain infectious factors, family factors, and inappropriate IYF practices.

In the Sabou health district, $72.9 \%$ of the households did not have a latrine (they used nature as their main toilet) and $63.8 \%$ did not have a rubbish bin. The National Survey on Food Insecurity and Malnutrition organized by the Ministry of Agriculture found that $80 \%$ of the households in the centre west did not have latrines (Olney et al., 2015). This considerable lack of latrines, coupled with inadequate waste management is believed to pollute surface waters, leading to the persistence of water-borne diseases. Access to safe drinking water is considered as one of the essential components of household nutrition in rural areas. If this access is not guaranteed, it results in individuals' exposure to many diseases, especially for young children.

In the Sabou health district, $21.8 \%$ of the households do not have access to drinking water for consumption, which results in consequences, especially among children under five years of age. A joint study carried out by $2 \mathrm{iE} / \mathrm{UNICEF}$ in 
suburb areas of the central plateau showed that $95 \%$ of the water consumed at homes was no longer drinkable because it was contaminated during the transport and storage chain (Bonkoungou, 2015). Poor water quality and inadequate hygiene practices are sources of diarrhoeal diseases and parasitic infections. Similarly, open defecation and littering on the streets have a negative impact on the health of communities, especially children.

Common illnesses encountered in children were malaria, diarrhoea, and respiratory infections with respective prevalence of $40.8 \%, 46.6 \%$, and $57.8 \%$ in the district, respectively. This high prevalence of morbidity is due to the high level of insalubrity in households caused by lack of latrines and garbage bins. This finding can also be explained by use of well water as a source of drinking water by households. The SMART survey of the Directorate of nutrition reported that the prevalence of diarrhoea and fever were $16.6 \%$ and $33.7 \%$ in the central-western region, respectively (Ministry of Health, 2016).

More than half of the households (68.8\%) were highly food insecure, $28.8 \%$ were moderately food insecure, and only $2.3 \%$ were low food insecure. Our results are similar to those found by the ministry of agriculture, which found that $64.4 \%$ of the households in the central-west were highly food insecure (Olney et al., 2015).

Today, the ideal of food security for all people at all times is far from being achieved in most households in Burkina Faso, where more than $20 \%$ of the population is undernourished (National Institute of Statistics and Demography (INSD ), 2010). This situation has been even worsened in recent years as the food and nutrition situation is threatened by the scarcity and poor distribution of rainfall.

The results showed a very low level of dietary diversity in most households at the district level. In the Sabou health district, $54.8 \%$ of the households had low dietary diversity (score $<4$ ), $36.4 \%$ had medium diversity, and only $8.7 \%$ had high dietary diversity. The staple foods for all households in rural areas are generally self- produced cereals and leafy vegetables.

Several factors are associated with acute malnutrition in children. Initially, the majority of malnourished children (77.4\%) were within the age range of 6-23 months, who showed high prevalence of acute malnutrition. So, this age group was considered as the preferred age for the onset of malnutrition (Victora et al., 2010, World Health Organization, 2005). Several factors can lead to the prevalence of malnutrition during 6 to 23 months of age including use of complementary foods since breast milk is no longer sufficient to cover the infant's needs. Malnutrition was associated to inadequate supplementary feeding in terms of quantity and quality.

In most children, illnesses associated with acute malnutrition include diarrhoea, malaria, and respiratory infections. These diseases are, directly or indirectly, the leading causes of death among young children in the developing countries (Organization, 2003). Almost all malnourished children, $96.8 \%$ had suffered from some kind of illness two weeks preceding the survey. Based on the results, $16.1 \%$ of the malnourished children had developed malaria, $29 \%$ suffered from diarrhoea, and $54.9 \%$ experienced a respiratory infection. These results are similar to those reported by other (Ouedraogo, 2013). He mentioned that the diseases associated with malnutrition were mainly diarrhoea (44\%) and respiratory infections (56\%). Zoungrana et al. reported that $18.4 \%$ of the malaria cases had acute malnutrition (Zoungrana et al., 2019), which confirms that morbidity caused by diarrhoea, malaria, and respiratory infections is a factor associated with acute malnutrition in children.

Household food insecurity and low dietary diversity were among other factors associated with malnutrition in children under five years of age. The prevalence of malnourish among children living in households with severe food insecurity and low dietary diversity were $80.6 \%$ and $61.3 \%$, respectively. This low dietary diversity accompanied by severe household food insecurity is found to be a factor associated with 
child malnutrition. The scarcity of food in households could be justified by the period of our survey, which was conducted from August 1 to August 30, when the various foodstuffs in the fields, on which the households directly depend, were not yet mature.

The IYF practices were very unsatisfactory among the malnourished children. Complementary feeding and diversification, i.e., consumption of at least 4 food groups in 24 hours, was also a factor associated with the nutritional status of children aged from 6 to 23 months. Considering the complementary feeding, no malnourished children received solid, semi-solid, or soft food the day before the survey. Only one malnourished child (3.2\%) consumed at least 4 food groups. The late onset of complementary feeding in these children can explain their nutritional status. Considering the high nutritional requirements of children within the age range of 6 to 23 months for rapid growth, appropriate complementary feeding would provide the key nutrients for good growth. Late food supplementation and early ablactation are thought to cause malnutrition.

\section{Conclusion}

Several factors are associated with acute malnutrition in children under five years of age in Burkina Faso. These factors included the age range of 6 to 23 months, morbidity, low dietary diversity accompanied by food insecurity, and some IYF practices, namely the inadequate introduction of complementary foods, and persistent low dietary diversity in children.

Preventive health and nutrition interventions have demonstrated that good IYF practices had a significant impact. Therefore, it is necessary to strengthen good IYF practices and to establish initiatives and programmes in favour of women to empower and educate them with regard to the integral growth of children.

\section{Conflicts of Interest}

The authors state no conflict of interest.

\section{Authors' contributions}

Savadogo A was involved in designing and supervising the study. Kabre $\mathrm{J}$ was involved in designing the study, collecting and analysing the data, as well as writing the manuscript. Bougma $\mathrm{S}$ and Zoungrana $\mathrm{B}$ participated in analysing the data and writing the manuscript. Traore $\mathrm{Y}$ supervised the study. All authors declare that they critically reviewed the manuscript and approved the final version submitted for publication.

\section{Acknowledgements}

The authors would like to thank the staff of the Sabou Health District and the CREN Maximilien COLBE for their multifaceted support during data collection.

\section{References}

Blanc AK \& Wardlaw T 2005. Monitoring low birth weight: an evaluation of international estimates and an updated estimation procedure. Bulletin of the World Health Organization. 83: 178-185.

Bonkoungou S 2015. Drinking water quality and related health risks in the South-West region: the case of the commune of Dano. International Institute for Water and Environment: Burkina Faso.

Carletto C, Zezza A \& Banerjee R 2013. Towards better measurement of household food security: Harmonizing indicators and the role of household surveys. Global Food Security. 2 (1): 30-40.

Ministry of Health 2014. National Protocol for the Integrated Management of Acute Malnutrition (PCIMA) in Bukina Faso.

Ministry of Health 2016. National Nutrition Survey. (ed. F. Report): Burkina Faso.

National Institute of Statistics and Demography (INSD ) 2010. Demographic and Health and Multiple Indicator Cluster Surveys (EDSBF-MICS IV). Preliminary report. Burkina Faso.

Olney DK, Pedehombga A, Ruel MT \& Dillon A 2015. A 2-year integrated agriculture and nutrition and health behavior change communication program targeted to women in Burkina Faso reduces anemia, wasting, and 
diarrhea in children 3-12.9 months of age at baseline: a cluster-randomized controlled trial. Journal of Nutrition. 145 (6): 1317-1324.

Organization WH 2003. Global strategy for infant and young child feeding. World Health Organization.

Ouedraogo A 2013. Outpatient follow-up of severely malnourished children from six to thirty-six months after hospitalization: case of CREN Morija in Ouagadougou. University of Ouagadougou: Burkina Faso.

Smith L \& Haddad L 2014. Reducing Child Undernutrition: Past Drivers and Priorities for the Post- MDG Era. IDS Working Papers. 2014 (441): 1-47.

UNICEF Staff 2011. The state of the world's children 2011-executive summary: Adolescence an age of opportunity.

UNICEF WHO 2000. Management of the child with a serious infection or severe malnutrition: guidelines for care at the first-referral level in developing countries.

Victora C, De Onis M, Hallal P, Blössner M \& Shrimpton R 2010. Worldwide timing of growth faltering: revisiting implications for interventions. Pediatrics. 125 (3): 473-480.

World Health Organization 2005. Guiding principles for feeding non-breastfed children 624 months of age.

Yousaf H, Zafar MI, Anjum F \& Adil SA 2018. Food security status and its determinants: A case of farmer and non-farmer rural households of the Punjab, Pakistan. Pakistan Journal of Agricultural Sciences. 55 (1).

Zoungrana B, et al. 2019. Performance and cost of management of severe acute malnutrition with complications in Kaya, Burkina Faso. Pan African Medical Journal. 34: 145-145. 